\section{On Energy Security of Smartphones}

${ }^{1,2}$ Xing Gao, ${ }^{1,2}$ Dachuan Liu, ${ }^{l}$ Daiping Liu and ${ }^{l}$ Haining Wang

${ }^{1}$ Electrical and Computer Engineering, University of Delaware, Newark, Delaware

${ }^{2}$ Computer Science, College of William and Mary, Williamsburg, Virginia

\section{Smartphone Energy Modeling} Energy energy modeling on each app:

- Breakdown the power consumption by every component and build energy models.

- A well designed battery interface to visualize energy consumption.

- High frequency and high accuracy.

- Existing energy malware is not hard to detect under the energy accounting and battery interface.

$\square$ However, existing energy modeling could be easily evaded!

\section{Attacking vectors}

- Wakelock- \& Screen-based Attack Vector. Screen energy profiling:

- 1. Take screen as an independent module.

- 2. Allocates screen energy to foreground app.

Problem: both mechanisms give malicious apps the possibility to deceive energy accounting.

Change the screen configuration in background.

\section{Defense}

* Architecture of E-Android

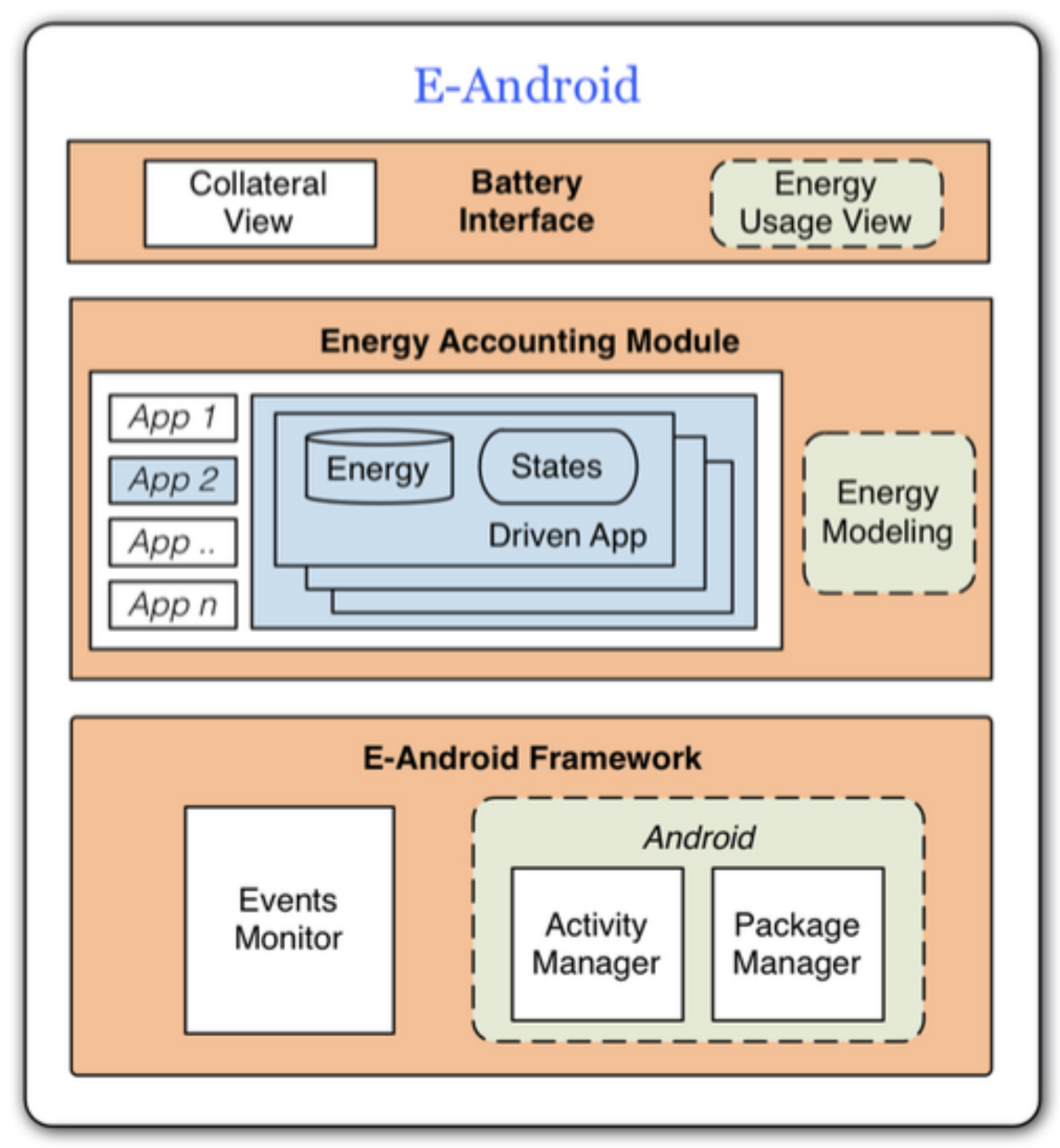

- Extension of Android framework.

- Record all events potentially invoke an energy collateral attack.

- Record the user ID of both apps as well as the type of the operation.

- Enhanced energy accounting module.

- Calculate energy consumption with consideration of collateral effects

- Revised battery interface.

- Inform users of all information related to energy consumption.

- Provide a detailed inventory specifying contribution of all attack related apps

\section{Attacking vectors}

* IPC-based Attack Vector.

$>$ Bob uses the Message app to film a 30s video.

$>$ A small camera window is embedded in the

Message. All occur in the Message.

$>$ It is reasonable to expect: the Message should be accounted for the energy consumption.

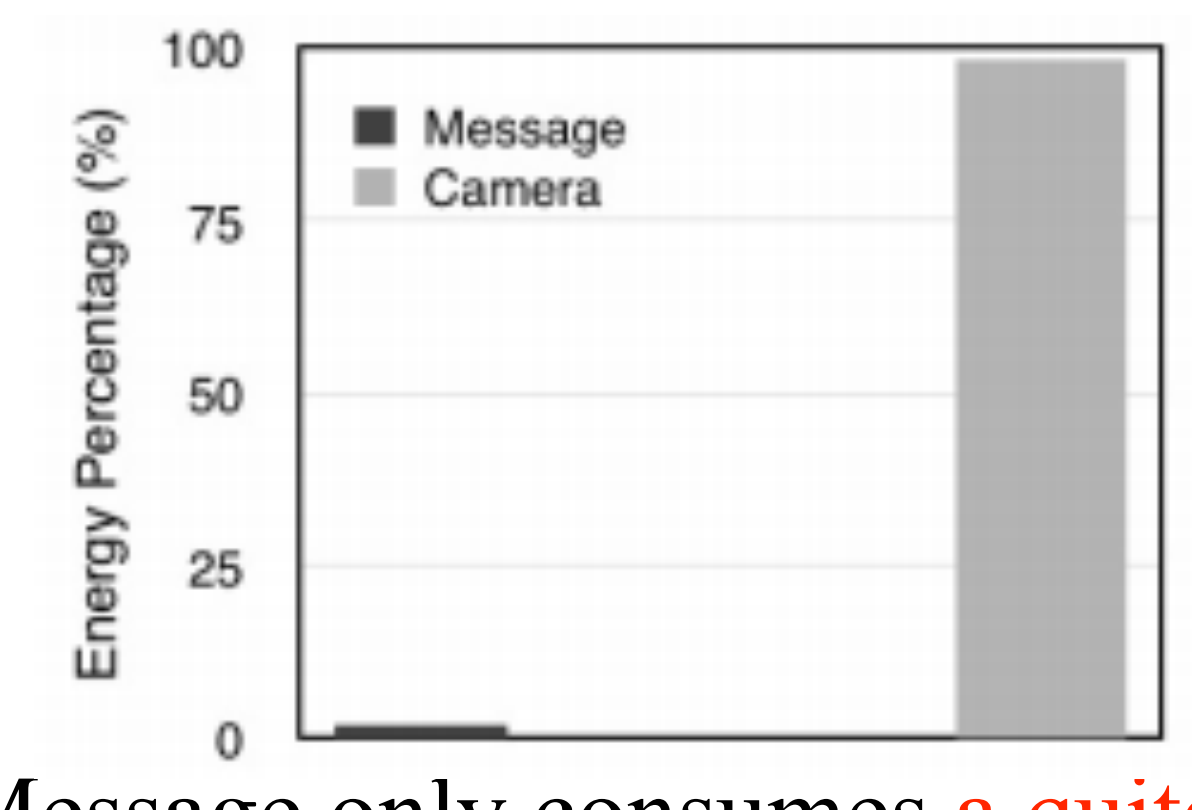

* Measured

by Android official app.

$>$ Message only consumes a quite small portion of energy.

$>$ The energy drained by video filming is assigned to the Camera.

$\square$ The Message sends an Intent to request the Camera app.

$\square$ It is the Camera app that actually records the video.

Problem: existing energy accounting modules overlook IPC.

\section{Energy Collateral Attacks}

\section{Attacks}

$>$ \#1 Hijack components belonging to other apps.

>\#2 When malware is launched by user, malware open other apps concurrently and make them run in background.

$>$ \#3 Bind to services without unbinding.

$>$ \#4 Interrupt attacked apps to background.

>\#5 Drain energy through changing screen configuration (such as escalating the brightness).

$>$ \#6 Acquire screen wakelock without releasing to keep screen on.

\section{$>$ Multi- \& Hybrid Attack.}

$>$ Multi attack on one app.

$>$ Energy attack chains.

\section{Attack Scenarios.}

- DoS attack.

- Mislead a user's attention to an innocent app.

- Unlike traditional attacks, target at reducing battery.

- Launch accompanying with traditional attacks.

\title{
Experiments
}

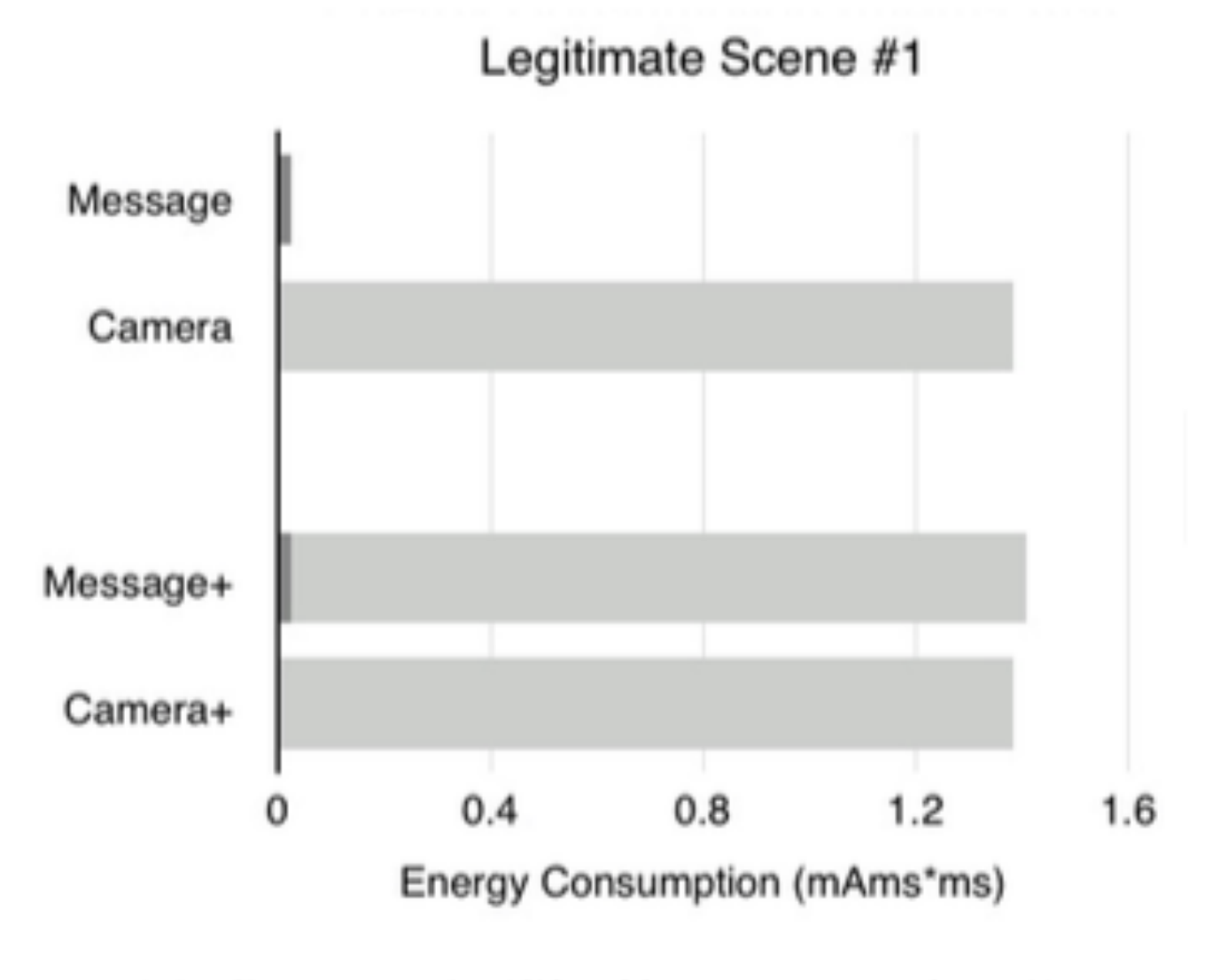

(a) Scene \#1 (Similar to attack \#1)

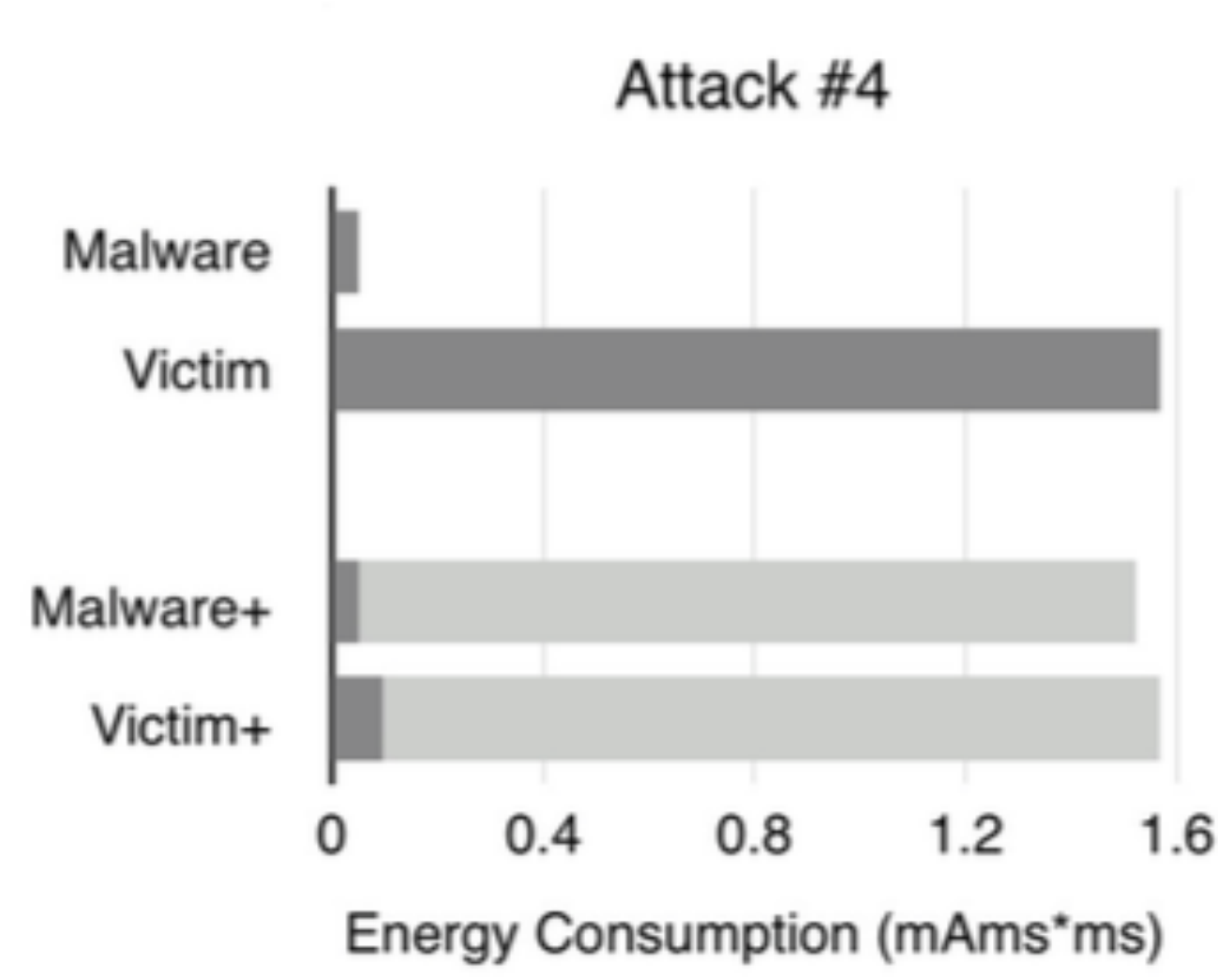

(d) Attack \#4

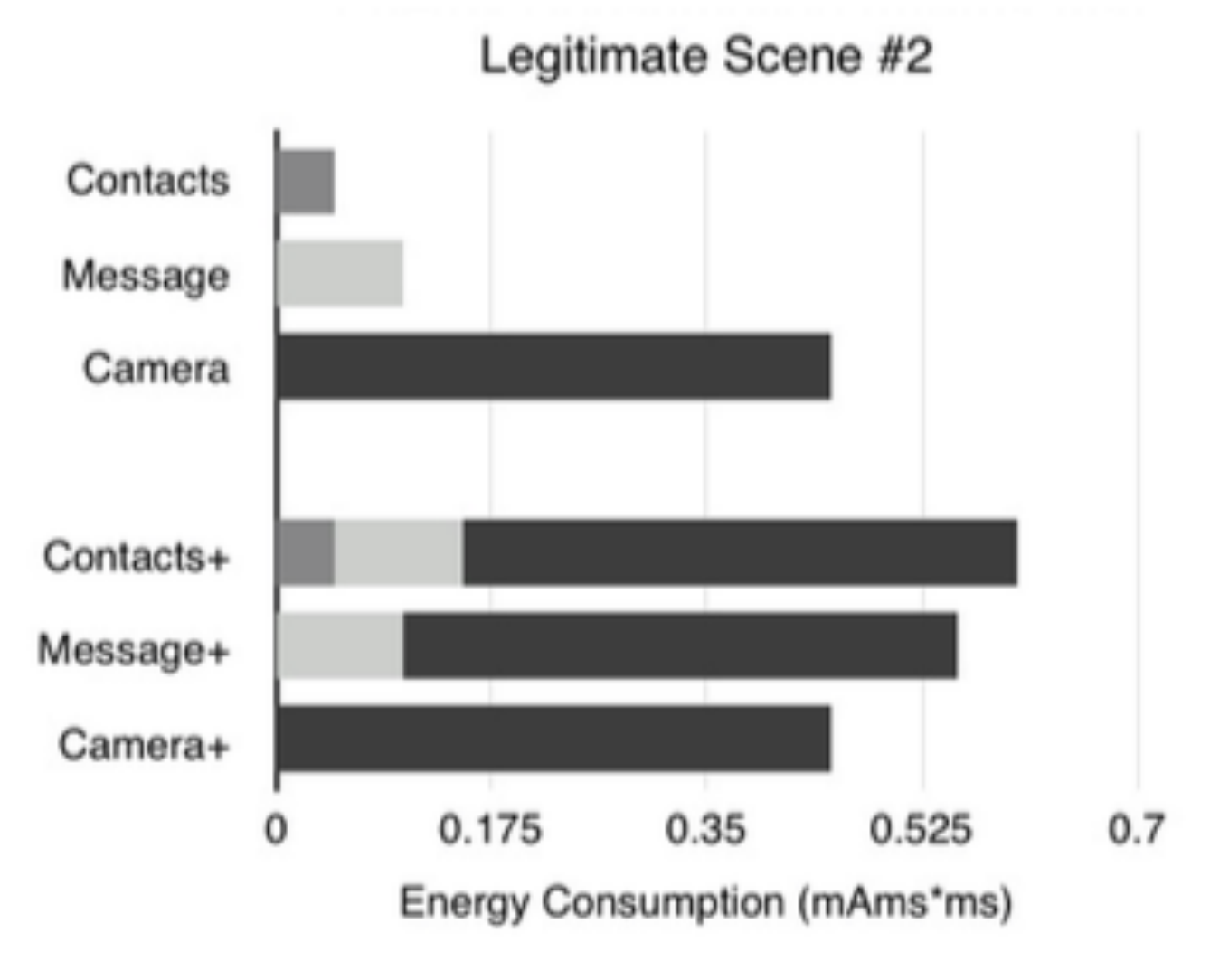

(b) Scene \#2 (Similar to hybrid attack)

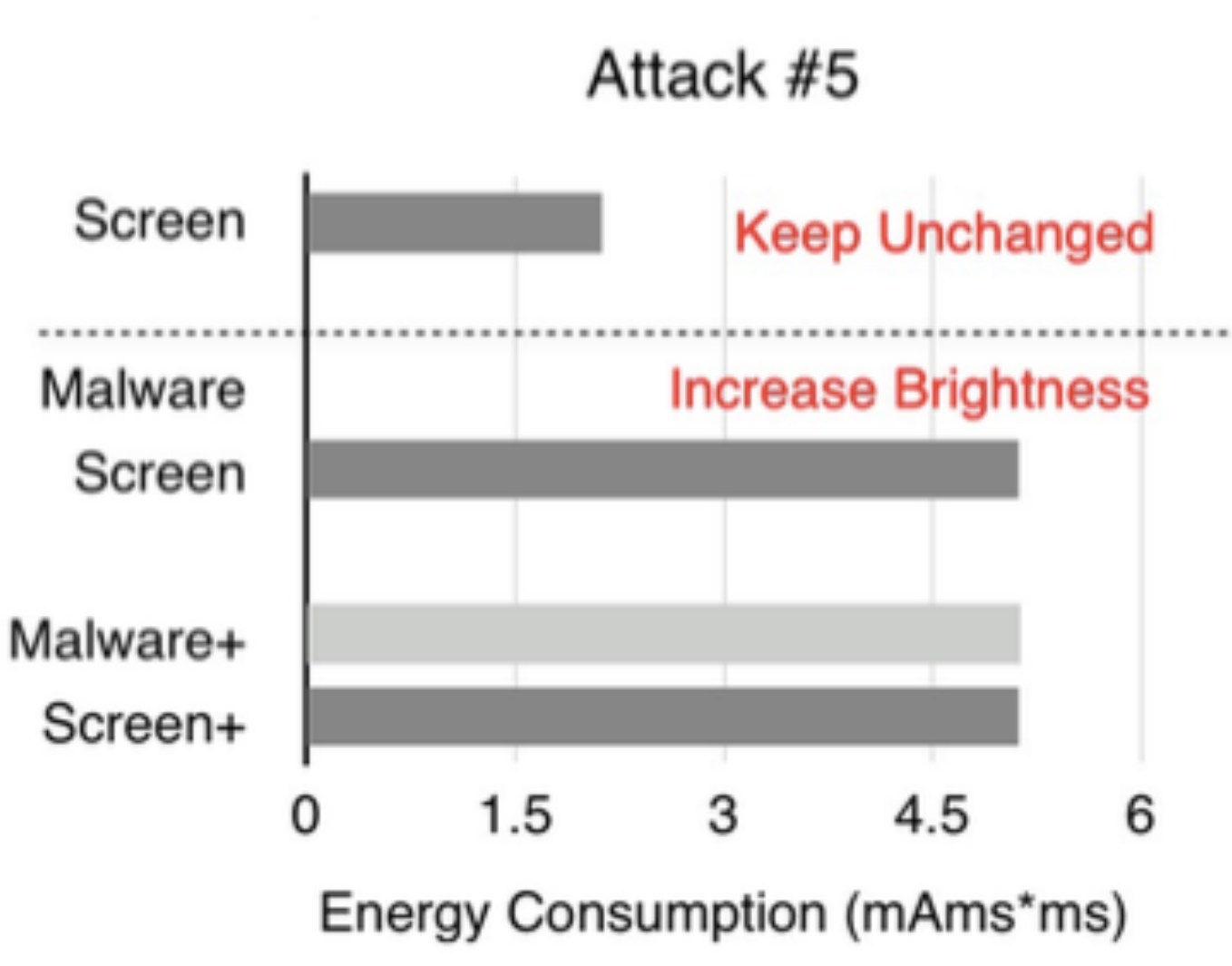

(e) Attack \#5

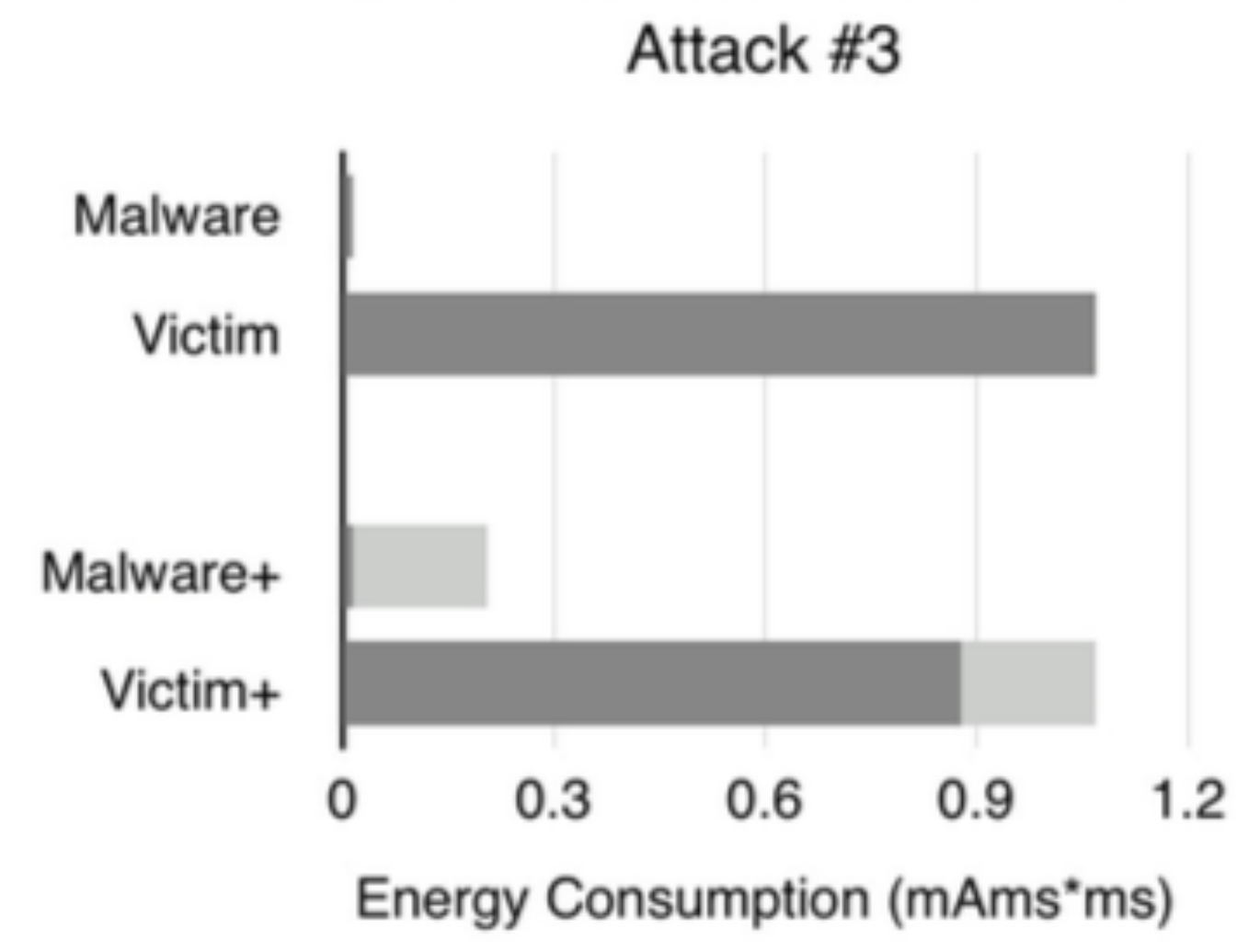

(c) Attack \#3

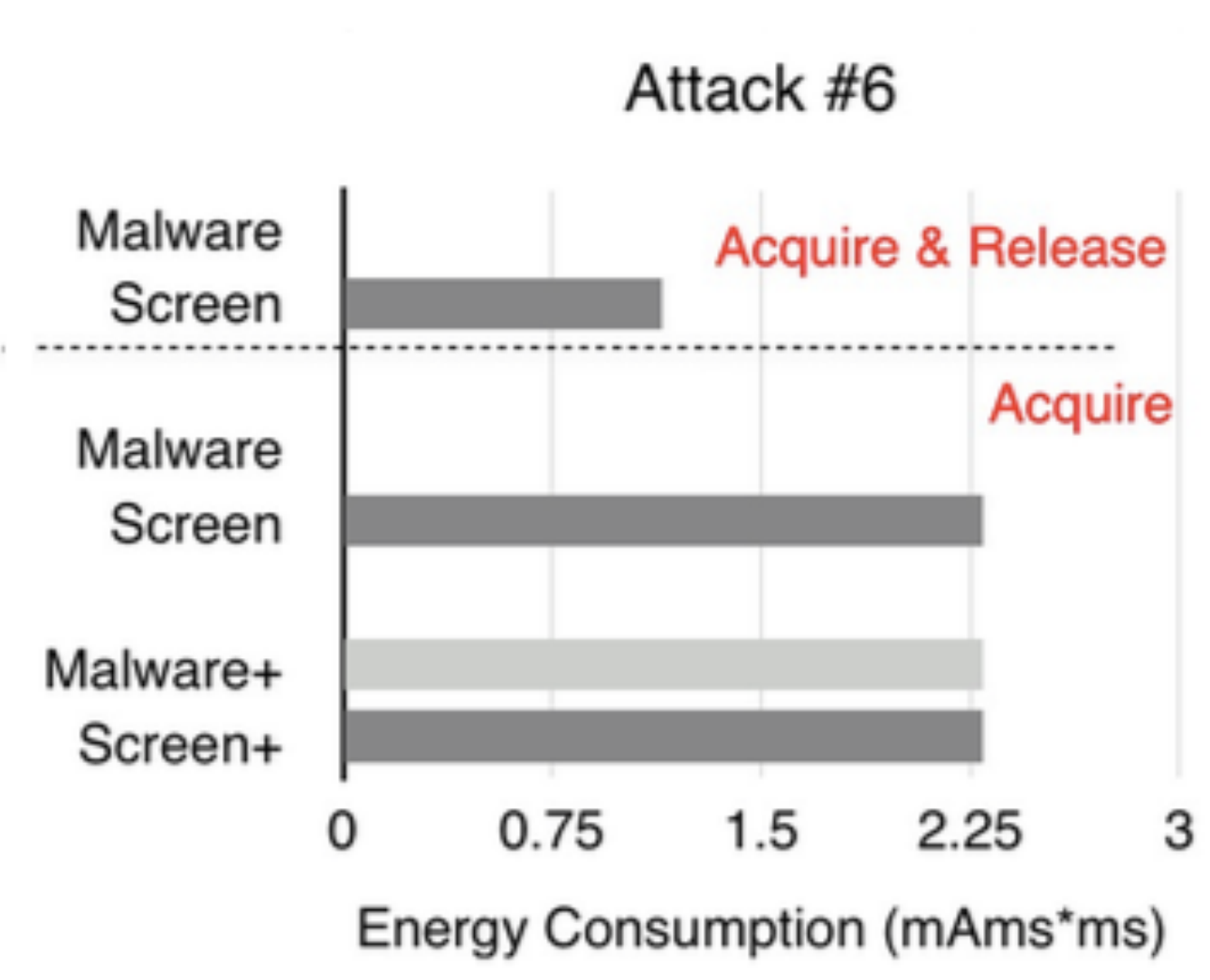

(f) Attack \#6

(a) Open the Message app 30s and then use it to take a 30s short video (Similar to the malware attack \#1). (b) Use the Contacts to open the Message, then films a 30s video (Energy attack chain).

* (c) Malware binds the victim's service and forces the service to run continuously after the attacked app attempts to stop the service.

(d) Interrupt the foreground app to background by detecting the quit dialog, using SurfaceLinger.

(e) Malware enhances brightness from service.

(f) Malware does not release screen wakelock.

$\square$ All those attacks could successfully bypass the supervision of the Android battery interface.

$\square$ E-Android can detect all these attacks. 